\title{
Immune-related hepatitis in a patient with hepatocellular carcinoma treated with nivolumab
}

\author{
Bernhard Scheiner · Matthias Pinter (D)
}

Received: 15 June 2021 / Accepted: 12 August 2021 / Published online: 9 September 2021

(C) The Author(s) 2021

\begin{abstract}
Summary We present a case of a male patient with advanced hepatocellular carcinoma who developed hepatic and dermatological immune-related adverse events during treatment with the immune checkpoint inhibitor nivolumab. We discuss relevant aspects regarding the management of immune-related hepatic adverse events, including the incidence and onset of the event, the requirement for immune-modulating medication, resuming of immunotherapy, and the association between the occurrence of immune-related adverse events and the outcome.
\end{abstract}

Keywords Immune-related hepatitis - Lichen ruber . Immunotherapy $\cdot$ Liver cancer

\section{Case presentation}

A 61-year-old male patient with alcoholic liver cirrhosis and a history of transjugular intrahepatic portosystemic shunt (TIPS) placement for refractory ascites underwent surveillance for hepatocellular carcinoma (HCC) with ultrasound at 6-month intervals at our institution. When ultrasound showed a new onset of portal vein thrombosis, the patient was referred for a computed tomography (CT) scan. The CT scan revealed a 6 -cm tumor with portal vein invasion, several smaller hypodense liver lesions, and no clear evidence of extrahepatic metastases. Biopsy finally confirmed the diagnosis of moderately differentiated HCC. The tumor marker alpha-fetoprotein was within the nor-

B. Scheiner, MD $\cdot$ M. Pinter, MD PhD $(\bowtie)$

Division of Gastroenterology and Hepatology, Department of Internal Medicine III, Medical University of Vienna, Waehringer Guertel 18-20, 1090 Vienna, Austria

Liver Cancer (HCC) Study Group Vienna, Medical University of Vienna, Vienna, Austria

matthias.pinter@meduniwien.ac.at mal range at this time. Based on a well-preserved liver function (Child-Pugh class A), a performance status of 0 (asymptomatic), and vascular tumor invasion, the patients was classified as having advanced-stage HCC (Barcelona Clinic Liver Cancer [BCLC] stage C).

After multidisciplinary team discussion, systemic treatment was recommended and the immune checkpoint inhibitor (ICI) nivolumab at a dose of $240 \mathrm{mg}$ every 2 weeks was initiated. After the second cycle, a grade 3 increase in transaminases was noted (Fig. 1). The patient was asymptomatic and had no signs of liver decompensation. Given the rapid increase, immune-related hepatitis (irHepatitis) was suspected. Nivolumab was paused and prednisolone was initiated with an intravenous bolus of $0.75 \mathrm{mg} / \mathrm{kg}$ followed by a maintenance dose of $50 \mathrm{mg}$ daily. After 1 week, the transaminase levels decreased significantly and prednisolone was tapered over a 4week course. However, after discontinuation of prednisolone, an increase in transaminases was noted again and prednisolone was reinitiated at a dose of $50 \mathrm{mg}$ daily for 2 weeks and then tapered over a course of 3 weeks. The transaminase levels eventually returned to normal values and nivolumab treatment was finally resumed around 3 months after initiation of steroids. Despite the 3-month interruption of nivolumab treatment, the patients achieved stable disease on follow-up imaging and remained stable for 2.5 years. He never developed an episode of irHepatitis again during this period.

However, approximately 1 year after the start of nivolumab treatment, the patient developed scaly, psoriasiform skin lesions on the upper and lower extremities (Fig. 2). After histological evaluation, the diagnosis of an immune-mediated verrucous lichen ruber was made. Given that the lichen was complicated by recurrent skin infections, the patient repeatedly received antibiotic treatment and nivolumab 


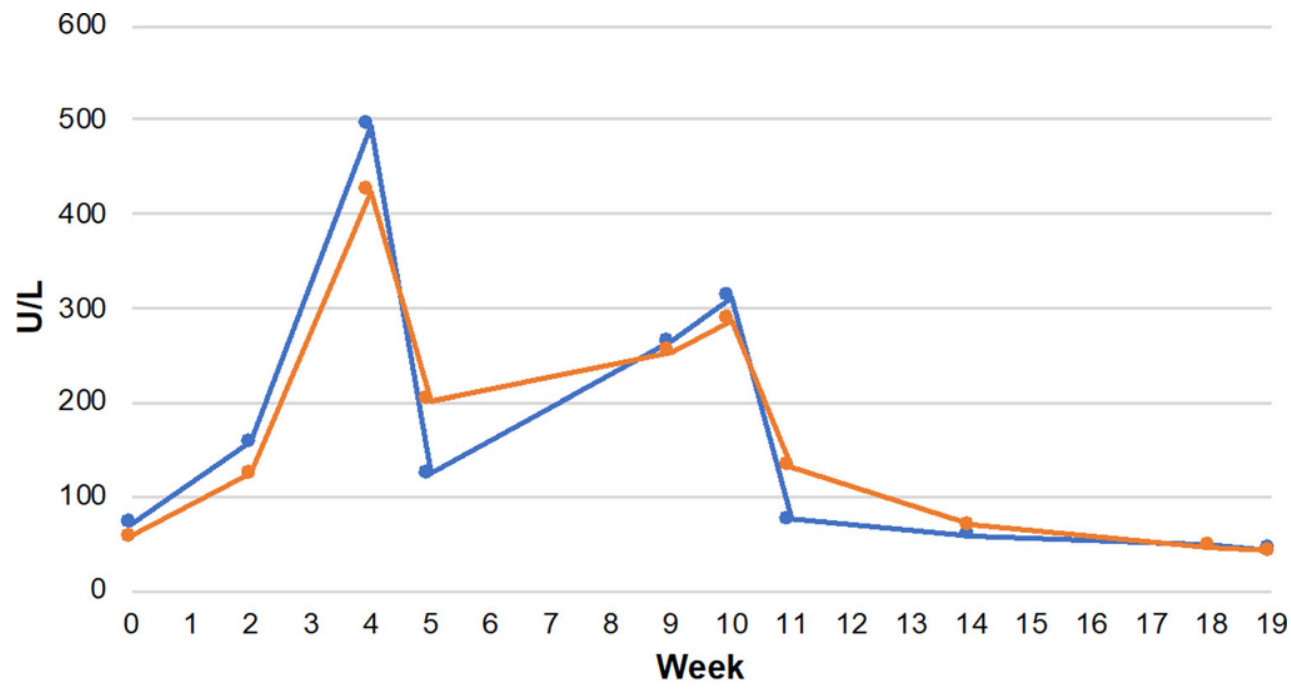

\section{$\rightarrow$ AST $\rightarrow$ ALT}

Fig. 1 Course of transaminases. At week 4 after initiation of nivolumab, the patient experienced a grade 3 increase in transaminases (upper limit of normal is $<50 \mathrm{U} / \mathrm{l}$ for both AST and ALT). Nivolumab was paused and treatment with prednisolone was initiated at a dose of $50 \mathrm{mg}$ daily. After steroid tapering, the transaminase levels increased again at week 9 , which prompted a new escalation of the prednisolone dose to $50 \mathrm{mg}$ daily for 2 weeks. Steroids were then tapered over a 3week course and transaminase levels eventually returned to normal values. Nivolumab was restarted at week 17. AST aspartate aminotransferase, $A L T$ alanine aminotransferase

Fig. 2 Lichenoid skin lesions. Around 1 year after initiation of nivolumab treatment, the patient developed scaly, psoriasiform skin lesions on the upper and lower extremities. The diagnosis of an immunemediated verrucous lichen ruber was made after histological evaluation
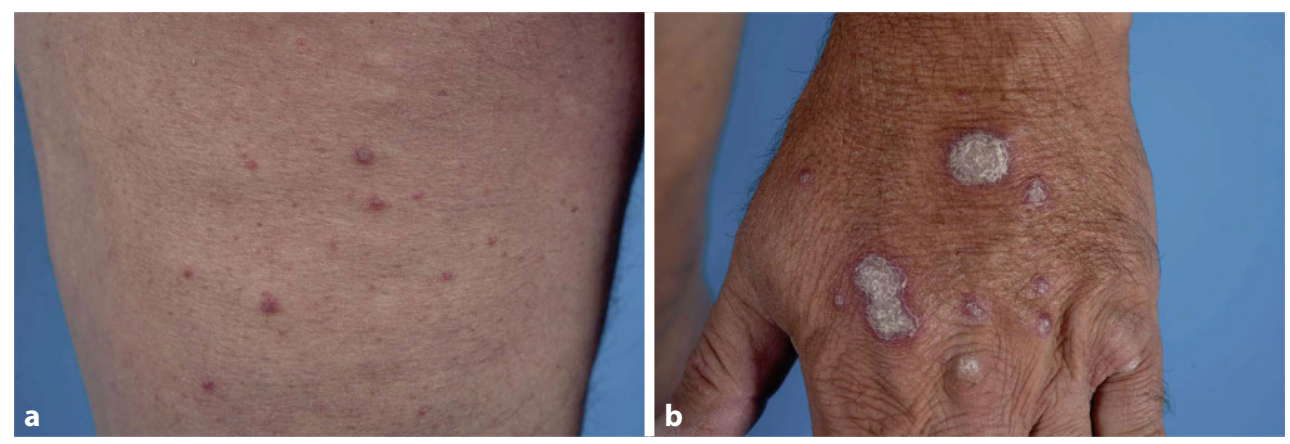

treatment was interrupted several times. The skin lesions were treated with topical steroids and photochemotherapy (PUVA).

Around 3 years after initiation, nivolumab was discontinued due to tumor progression. Thereafter, the patient received several lines of systemic therapy, including sorafenib, lenvatinib, cabozantinib, and regorafenib, and died roughly 1 year later due to further tumor progression.

\section{Discussion}

Several systemic therapies are approved for the treatment of patients with HCC, including sorafenib and lenvatinib in first-line treatment, and regorafenib, cabozantinib, and ramucirumab in patients pretreated with sorafenib. Immunotherapy has been added to the treatment armamentarium only recently [1]. The immune checkpoint inhibitors nivolumab, pembrolizumab, and the combination of nivolumab plus ipilimumab have been conditionally approved based on promising data from phase II trials in the United States (but not in Europe; [1, 2]). The combination of atezolizumab and bevacizumab improved overall survival and progression-free survival over sorafenib in a phase III trial [3], and represents the new reference standard in systemic front-line therapy of HCC [1].

Immune-related adverse events of immune checkpoint inhibitors can affect several organs, including the liver. Immune-related hepatic adverse events usually occur within 4-12 weeks of immunotherapy initiation. They affect approximately $5-10 \%$ of patients treated with monotherapy, but are much more frequent upon combination of ICIs (15-30\%). During diagnostic work-up, differential diagnoses (i.e., intrahepatic tumor progression, hepatotoxic medication, acute viral infection or flares in patients with chronic 
viral hepatitis, alcohol abuse, thrombotic events or benign biliary obstructions) need to be excluded [4-7]. Notably, in patients developing an increase in liver enzymes during ICI treatment, immune-related hepatic adverse events were diagnosed in only $16 \%$ of cases, while around half of the patients had tumor progression [8].

In our patient, a grade 3 increase in transaminase levels developed 4 weeks after nivolumab initiation. Given the rapid onset and the low likelihood of other potential causes, we suspected irHepatitis. The good response to steroid treatment eventually confirmed the diagnosis. The adverse event was resolved around 3 months after steroid initiation. This is well in line with previous reports on the management of immunerelated hepatic adverse events in patients with HCC receiving nivolumab [9]. Of 37 patients experiencing hepatic adverse events, seven (19\%) required systemic corticosteroids and only one patient (3\%) required additional mycophenolic acid. Resolution of the event occurred in all seven patients (100\%) requiring immune-modulating medication after a median time of 19.4 weeks [9].

According to current recommendations, PD-(L)1targeted ICIs can be resumed in cases of grade 3 irHepatitis while permanent discontinuation is recommended in patients experiencing grade 4 irHepatitis [4]. In our patient, nivolumab was resumed when steroids were discontinued and transaminases returned to baseline values. Even though our patient received nivolumab for more than 2 years, he never developed an episode of irHepatitis again. However, the further course of treatment was complicated by lichen ruber, a known but rather rare dermatological adverse event during ICI treatment, [10] that occurred approximately 1 year after nivolumab initiation.

The occurrence of immune-related adverse events may be associated with improved outcomes in cancer patients treated with ICIs [11-13], and this association could be even more pronounced in patients with multisystem immune-related adverse events [14]. Our patient, who experienced two immune-related adverse events, failed to respond to treatment but achieved stable disease for over 2 years, which can be considered treatment success.

\section{Conclusion}

Immune-related hepatic adverse events usually occur early after ICI initiation, sometimes require systemic corticosteroids, but rarely additional immune-modulating medication, and mostly resolve within several weeks of initiation of immunomodulators [4, 9].

Funding Open access funding provided by Medical University of Vienna.

Conflict of interest B. Scheiner received travel support from AbbVie, Ipsen and Gilead. M. Pinter is an investigator for Bayer, BMS, and Lilly; he received speaker honoraria from
Bayer, BMS, Eisai, and MSD; he is a consultant for Bayer, BMS, Ipsen, Eisai, Lilly, and MSD, and he received travel support from Bayer and BMS.

Open Access This article is licensed under a Creative Commons Attribution 4.0 International License, which permits use, sharing, adaptation, distribution and reproduction in any medium or format, as long as you give appropriate credit to the original author(s) and the source, provide a link to the Creative Commons licence, and indicate if changes were made. The images or other third party material in this article are included in the article's Creative Commons licence, unless indicated otherwise in a credit line to the material. If material is not included in the article's Creative Commons licence and your intended use is not permitted by statutory regulation or exceeds the permitted use, you will need to obtain permission directly from the copyright holder. To view a copy of this licence, visit http://creativecommons.org/licenses/by/4.0/.

\section{References}

1. Bruix J, Chan SL, Galle PR, et al. Systemic treatment of hepatocellular carcinoma: an EASL position paper. J Hepatol. 2021; https://doi.org/10.1016/j.jhep.2021.07. 004.

2. Pinter M, Jain RK, Duda DG. The current landscape of immunecheckpointblockadein hepatocellularcarcinoma: a review. JAMAOncol. 2021;7:113-23.

3. Finn RS, Qin S, Ikeda M, et al. Atezolizumab plus bevacizumab in unresectable hepatocellular carcinoma. N Engl JMed. 2020;382:1894-905.

4. Sangro B, Chan SL, Meyer T, et al. Diagnosis and management of toxicities of immune checkpoint inhibitors in hepatocellular carcinoma. J Hepatol. 2020;72:320-41.

5. Haanen J, Carbonnel F, Robert C, et al. Management of toxicities from immunotherapy: ESMO clinical practice guidelines for diagnosis, treatment and follow-up. Ann Oncol. 2017;28:iv119-iv42.

6. Boutros C, Tarhini A, Routier E, et al. Safety profiles of anti-CTLA-4 and anti-PD-1 antibodies alone and in combination. Nat Rev Clin Oncol. 2016;13:473-86.

7. Hassel JC, Heinzerling L, Aberle J, et al. Combined immune checkpoint blockade (anti-PD-1/anti-CTLA-4): evaluation and management of adverse drug reactions. Cancer Treat Rev. 2017;57:36-49.

8. Cunningham M, Iafolla M, Kanjanapan Y, etal. PS-139-Liver enzyme elevations and hepatotoxicity in patients treated with checkpoint inhibitor immunotherapy. J Hepatol. 2019;70(1):e89.

9. Julien K, Leung HT, Fuertes C, et al. Nivolumab in advanced hepatocellular carcinoma: safety profile and select treatment-related adverse events from the checkmate 040 study. Oncologist. 2020;25:e1532-e40.

10. Wongvibulsin S, Pahalyants V, Kalinich M, et al. Epidemiology and risk factors for the development of cutaneous toxicities in patients treated with immune-checkpoint inhibitors: a United States population-level analysis. J Am Acad Dermatol. 2021; https://doi.org/10.1016/j.jaad.2021. 03.094.

11. Haratani K, Hayashi H, Chiba Y, et al. Association of immune-related adverse events with nivolumab efficacy in non-small-cell lung cancer. JAMAOncol. 2018;4:374-8.

12. Maher VE, Fernandes LL, Weinstock C, et al. Analysis of the association between adverse events and outcome in patients receiving a programmed death protein 1 or programmed death ligand 1 antibody. J Clin Oncol. 2019;37:2730-7. 
13. Das S, Johnson DB. Immune-related adverse events and anti-tumor efficacy of immune checkpoint inhibitors. J Immunother Cancer. 2019;7:306.

14. Shankar B, Zhang J, Naqash AR, et al. Multisystem immunerelated adverse events associated with immune checkpoint inhibitors for treatment of non-small cell lung cancer. JAMA Oncol. 2020;6:1952-6.

Publisher's Note Springer Nature remains neutral with regard to jurisdictional claims in published maps and institutional affiliations. 